\title{
Leukemia survival in children, adolescents, and young adults: influence of socioeconomic status and other demographic factors
}

\author{
Erin E. Kent · Leonard S. Sender · Joan A. Largent • \\ Hoda Anton-Culver
}

Received: 2 January 2009/ Accepted: 11 May 2009/Published online: 4 June 2009

(c) The Author(s) 2009. This article is published with open access at Springerlink.com

\begin{abstract}
Objective In California, leukemia represents $\sim 35,5$, and $2 \%$ of all cancers in children (aged 0-14), adolescents (1529), and young adults (30-39), respectively. Poorer survival has been previously noted in individuals residing in lower socioeconomic status (SES) neighborhoods. We explored the relationship between SES and survival as modified by age and race/ethnicity using data from the California Cancer Registry.

Methods A total of 7,688 incident cases of first primary leukemia diagnosed during 1996-2005 in individuals aged 0-39 at diagnosis were included in this study. Univariate analyses of overall survival were conducted using the Kaplan-Meier method and multivariate survival analyses were performed using Cox proportional hazard regression to estimate hazard ratios.

Results Multivariate analyses showed that overall survival and lymphoid cancer-specific survival was reduced in those individuals aged 15-39 compared to children aged 0-14. Although shorter survival was observed in nonwhites, an association between lower-SES neighborhood
\end{abstract}

E. E. Kent $(\bowtie) \cdot$ J. A. Largent · H. Anton-Culver Department of Epidemiology, University of California, Irvine, 224 Irvine Hall, Irvine, CA 92697-7555, USA

e-mail: ebardin@uci.edu

\section{S. Sender}

Chao Family Comprehensive Cancer Center,

University of California, Irvine, Irvine, CA, USA

L. S. Sender

CHOC Children's Hospital, Orange, CA, USA

J. A. Largent - H. Anton-Culver

Genetic Epidemiology Research Institute,

University of California, Irvine, Irvine, CA, USA and shorter survival was significant only for non-Hispanic whites (NHWs) ( $p$ value for trend $<0.05$ ). Lack of insurance was significantly associated with shorter survival for all race/ethnicities examined except Asian/Pacific Islanders ( $p$ value $<0.05)$.

Conclusion Lower survival in individuals diagnosed with leukemia was observed in adolescents and young adults compared to children and in non-whites compared to NHWs. Further, the independent effects on survival of both low SES and lack of insurance at diagnosis persisted after adjustment for demographic variables and varied across race/ethnicities.

Keywords Leukemia - Survival analysis . Adolescent and young adult - Socioeconomic

\section{Introduction}

In California, leukemia represents $\sim 34.9,4.9$, and $2.4 \%$ of all cancers in individuals aged 0-14, 15-29, and 30-39, respectively. For adolescents aged 15-19, leukemia is the fourth leading type of cancer, with $\sim 746$ new cases diagnosed each year in California [1].

Marked differences in cancer incidence and survival by race/ethnicity persist. Although non-Hispanic whites (NHWs) have the highest overall cancer incidence rate, this group also experiences the highest overall cancer survival rate [2-4]. In California, Hispanics/Latinos are disproportionately affected in certain cancers. Hispanics aged 15-19 have twice the incidence rate of leukemia than NHWs $[1,3]$ and experience a survival rate at least $7 \%$ lower than NHWs [3].

The rapid progression of acute leukemias may present challenges in accessing care when compared to other 
cancer types, particularly for under-resourced individuals. Access to clinical trials [5], availability of quality treatment [6], availability of support systems [7], and having adequate health insurance [8] have all been shown to be positively associated with neighborhood socioeconomic status (nSES) and survival, and nSES may mediate the race/ethnicity survival relationship [9, 10]. Further, financial considerations may influence an individual's decision to seek and maintain cancer treatment [11].

Although adolescents and young adults (AYAs) have been variably defined, the National Cancer Institute prefers the inclusive age span of 15-39. Using this designation, leukemia represents the third most common cancer in AYAs and the most common cancer type in younger children [12]. Leukemia survival is improving for AYAs [13], but survival remains worse for AYAs than for children $[14,15]$, and little attention has been paid to possible socioeconomic disparities in survival among these age groups. The objectives of the present study were to examine the relative influences of neighborhood socioeconomic status as modified by age at diagnosis, race/ ethnicity, and insurance status at diagnosis on overall survival and leukemia-specific survival using populationbased cancer registry data.

\section{Patients and methods}

\section{Study population}

We performed a retrospective case-only analysis of all leukemia cases diagnosed between the age of 0 and 39 years in California from 1996 to 2005 using the California Cancer Registry (CCR) database $(n=7,688) .{ }^{1}$ The CCR is part of the National Cancer Institute's Surveillance, Epidemiology and End Results (SEER) program, with standardized data collection and quality control protocols in place since 1988 [16-19]. Both case reporting and follow-up completion rates are higher than $95 \%$ for the entire state of California [20]. After data are abstracted from medical and laboratory records by trained tumor registrars [18], tumor site and histology are coded according to the World Health Organization's criteria in International Classification of Diseases for Oncology (ICD-O, 3rd edition) [21].

For this analysis, patient cases were selected based on histologic types for leukemias (SEER primary site codes 35000-35999) according to ICD-O-3: acute lymphocytic leukemia (ALL; 9800, 9801, 9820, 9827, 9831-9834),

\footnotetext{
1 This study was approved by the University of California, Irvine Institutional Review Board (IRB) under the exempt category status (IRB\# 2008-6236).
}

acute myeloid leukemia (AML; 9805, 9860, 9870, 9891, 9930, 9931, 9940, 9948, 9964), chronic myeloid leukemia (CML; 9863, 9875, 9876, 9945, 9946, 9963), and all other morphologies (other; 9733). Although chronic lymphocytic leukemia (CLL; 9823) is considered one of the four primary types of leukemia, it is commonly seen only in patients over the age of 65 and there were only 89 diagnoses $(<0.01 \%)$ that fit our eligibility criteria. Therefore, CLL cases were excluded from all subsequent analyses beyond descriptive statistics.

Analyses were conducted on cases diagnosed between 1996 and 2005. Type of reporting source was available for each case, with 33 cases identified only through death certificate and nine cases identified through autopsy alone. The remaining cases were identified through high-quality reporting sources (hospital, inpatient/outpatient centers, oncology treatment centers, laboratories, or private practitioners). The CCR adheres to SEER standards, which require annual follow-up of patients to ensure that survival rates are as accurate as possible [18]. The registrars use both passive follow-up methods (annual record linkages with sources including the California State Death File, National Death Index, Social-Security Death Master File, Medicare, and Medicaid) as well as active follow-up (contact with physicians' offices, hospitals, and patient families). The observation period ended at the end of 2005 because for this dataset, cause of death could only be verified by death certificate for cases deceased before the end of 2005.

Recorded data included age at diagnosis, demographic information, histology, treatment during the first course of therapy, nSES, vital status, treatment hospital type (pediatric or otherwise), and insurance status. Race and ethnicity (Hispanic/Latino or not) are abstracted from patients' medical records following the coding procedures outlined by the CCR [18]. The nSES variable used in the CCR is a single index created from statewide census data measures of education, income, and occupation, previously described [22]. The variable was created from a principle component analysis of census block group-level data, including median educational attainment, median household income, proportion below $200 \%$ of the federal poverty level, median house value, median rent, percent employed, and proportion of the population with blue-collar employment. Quintiles for the nSES score were included for analysis.

Cause of death

Cause of death was recorded according to the International Classification of Disease (ICD) criteria in effect at the time of death [21], using the ICD-9 codes for deaths prior to 2000 and ICD-10 codes for deaths in 2000 and later. Hospital registrars contact cases annually and CCR staff review state death certificates on an annual basis to identify 
deceased registry patient cases. The last date of follow-up was either the date of death or the last date of contact. Lymphoid and hematopoietic cancer-specific deaths included codes 204.0-208.9 and C810-C959 [23].

\section{Statistical analyses}

Demographic characteristics and clinical parameters were analyzed using Pearson's $\chi^{2}$ test and analysis of variance (ANOVA) for comparing continuous variables across more than two groups. Life tables and Kaplan-Meier curves were generated for age group, race/ethnicity, nSES categories, and insurance status and were compared with the log-rank test. Cox proportional hazard regression was performed to generate adjusted hazard ratios (HRs) for overall survival (OS) and lymphoid/hematopoietic cancer-specific survival (LSS) using SAS 9.1 (SAS Institute, Inc., Cary, NC), controlling for race/ethnicity, leukemia type, nSES, insurance status, gender, and year of diagnosis. Analyses stratified by age group (0-14, 15-29, and 30-39), race/ethnicity [NHW, non-Hispanic black (NHB), Hispanic/Latino (HL), Asian/ Pacific Islander (API)], and leukemia type (ALL, AML, CML) were also conducted to investigate effect modification on any nSES effects on survival. Treatment hospital type (pediatric, pediatric affiliate, or other) was included for the stratified age-group analysis. Statistical significance was assumed for a two-tailed $p=0.05$.

\section{Results}

\section{Demographic data}

Of the 7,688 incident first primary leukemias diagnosed from 1996 to 2005 and included in the analysis, there were 2,689 NHWs, 389 NHBs, 3,760 HLs, and 778 APIs. Demographic data for the entire patient population are presented in Table 1. There was significant variation in diagnostic types by age group, with children aged 0-14 accounting for the majority of ALL $(3,409,72.6 \%$, $p<0.0001$ ), a fairly even distribution among age groups for AML, and young adults aged 30-39 comprising the majority of CLL $(79,88.8 \%, p<0.0001)$, and CML $(421,55.0 \%$, $p<0.0001)$ cases. Hispanics/Latinos comprised the largest number of ALL cases $(2,542,54.1 \%, p<0.0001)$, while NHWs accounted for the most cases in all other subtypes. Overall and for each individual subtype, the number of male cases outnumbered female cases $(p<0.0001)$ (Table 1).

Univariate analysis

Kaplan-Meier survival curves for overall survival by age group, race/ethnicity, nSES, and insurance status were generated (Fig. 1). Overall univariate survival was lower in AYAs than in children $(p<0.001)$, non-whites than in NHWs $(p<0.001)$, in lower nSES groups than in higher ones $(p<0.001)$, and in those without insurance or with unknown insurance status at diagnosis compared to those with any insurance $(p<0.001)$. Figure 2 shows 5 -year survival rates by age at diagnosis. Excluding infant leukemias (aged $0-1$; believed to have a different etiologic profile from leukemias that develop later in life [24]), survival rates appear to be higher in children. From age five to $\sim 15-16$, survival rates and overall survival times decline with some leveling off in older individuals.

Neighborhood socioeconomic and insurance status analysis

Significant distributional variation $(p<0.001)$ among the five nSES quintiles for different race/ethnicities exists: $\sim 26.9 \%$ of NHWs and $27.8 \%$ of APIs but only $5.2 \%$ of HLs and $6.0 \%$ of NHBs belong to the highest quintile. Conversely, $45.5 \%$ of HLs and $23.6 \%$ of NHBs but only $8.3 \%$ of NHWs and $12.1 \%$ of APIs belong to the poorest nSES quintile. Approximately $45.5 \%$ of uninsured cases and $38.7 \%$ of cases with unknown insurance status resided in one of the lowest nSES quintile areas, compared to only $26.0 \%$ of cases with any insurance $(p<0.001)$. On the contrary, only $7.3 \%$ of the uninsured and $9.7 \%$ of those with unknown insurance status lived in the highest nSES quintile area at diagnosis, compared to $16.2 \%$ of cases with any of type insurance.

Insurance status rates by race/ethnicity and 5-year age at diagnosis groups were examined. Hispanics/Latinos had the highest proportion of being uninsured at diagnosis (HLs: $6.5 \%$, NHBs: $3.5 \%$, APIs: $3.1 \%$, NHWs: $1.8 \%$ ) with statistically significant differences among race/ethnic groups $(p<0.001)$. There was also a sharp rise in the proportion of uninsured from individuals aged 15-19 (6.6\%) to those aged 20-24 (11.0\%), possibly explained by the stipulations of most policies permitting minors to be covered by parental insurance policies, as well as college insurance plans. For subsequent multivariate survival analyses, individuals with unknown insurance status were combined with uninsured cases, as the univariate survival curves for these two groups appeared to overlap (Fig. 1).

Cause of death analysis

Verified cause of death was available for patients through 2005. Of the 7,688 cases of leukemia examined for these analyses, 2,194 had died by the end of 2005. Of those deaths, $1,990(90.7 \%)$ were due to neoplasms of the lymphoid or hematopoietic tissue and $17(0.8 \%)$ were the result of other cancer-related causes. As of this analysis, cause of 
Table 1 Demographic information and incidence for the major leukemia subtypes, for first primary diagnoses

\begin{tabular}{|c|c|c|c|c|c|c|}
\hline Characteristic & $\begin{array}{l}\text { Acute } \\
\text { lymphocytic } \\
n=4,695 \\
n(\%)\end{array}$ & $\begin{array}{l}\text { Acute } \\
\text { myeloid } \\
n=2,137 \\
n(\%)\end{array}$ & $\begin{array}{l}\text { Chronic } \\
\text { lymphocytic } \\
n=89 \\
n(\%)\end{array}$ & $\begin{array}{l}\text { Chronic } \\
\text { myeloid } \\
n=765 \\
n(\%)\end{array}$ & $\begin{array}{l}\text { Total } \\
n=7,688 \\
n(\%)\end{array}$ & $p$ \\
\hline \multicolumn{7}{|l|}{ Age at diagnosis } \\
\hline $0-14$ & $3,409(72.6)$ & 675 (31.6) & $2(2.3)$ & $72(9.4)$ & $4,158(54.1)$ & \multirow[t]{3}{*}{$<0.0001$} \\
\hline $15-29$ & 877 (18.7) & $700(32.8)$ & $8(9.0)$ & $272(35.6)$ & $1,857(24.2)$ & \\
\hline $30-39$ & $409(8.7)$ & $762(35.7)$ & $79(88.8)$ & $421(55.0)$ & $1,673(21.8)$ & \\
\hline Mean age (SD) & $10.6(10.1)$ & $21.6(12.6)$ & $34.5(6.3)$ & $28.1(9.5)$ & $15.7(12.7)$ & $<0.0001$ \\
\hline \multicolumn{7}{|l|}{ Year of diagnosis } \\
\hline 1996-1999 & $1,811(38.6)$ & $881(28.9)$ & $34(38.2)$ & $328(42.9)$ & $3,054(39.7)$ & \multirow[t]{3}{*}{0.035} \\
\hline $2000-2003$ & $1,889(40.2)$ & $848(27.5)$ & $42(47.2)$ & 307 (9.9) & $3,087(40.2)$ & \\
\hline 2004-2005 & $995(21.2)$ & $408(19.1)$ & 13 (14.6) & $130(17.0)$ & $1,547(20.1)$ & \\
\hline \multicolumn{7}{|l|}{ Gender } \\
\hline Male & $2,747(58.5)$ & $1,155(54.1)$ & $495(64.7)$ & $56(62.9)$ & 4,454 (57.9) & \multirow[t]{2}{*}{$<0.0001$} \\
\hline Female & $1,948(41.5)$ & $982(46.0)$ & $270(35.3)$ & $33(37.1)$ & $3,234(42.1)$ & \\
\hline \multicolumn{7}{|l|}{ Race/ethnicity } \\
\hline Non-Hispanic white & $1,529(32.6)$ & $810(37.9)$ & $50(56.2)$ & $300(39.2)$ & $2,689(35.0)$ & \multirow[t]{5}{*}{$<0.0001$} \\
\hline Non-Hispanic black & $178(3.8)$ & $149(7.0)$ & $7(7.9)$ & $54(7.1)$ & $389(5.1)$ & \\
\hline Hispanic/Latino & $2,542(54.1)$ & $915(42.8)$ & $16(18.0)$ & $287(37.5)$ & $3,760(48.9)$ & \\
\hline Asian/Pacific Islander & $408(8.7)$ & $252(11.8)$ & $7(7.9)$ & $110(14.4)$ & $778(10.1)$ & \\
\hline Other & $38(0.8)$ & $11(0.5)$ & $9(10.1)$ & $14(1.8)$ & $72(0.9)$ & \\
\hline \multicolumn{7}{|l|}{ nSES } \\
\hline Highest & $721(15.4)$ & $319(14.9)$ & $24(27.0)$ & $125(16.3)$ & $1,189(15.5)$ & \multirow[t]{5}{*}{$<0.0001$} \\
\hline High & $768(16.4)$ & $381(17.8)$ & $20(22.5)$ & $157(20.5)$ & $1,327(17.3)$ & \\
\hline Middle & $830(17.7)$ & $415(19.4)$ & $20(22.5)$ & $156(20.4)$ & $1,421(18.5)$ & \\
\hline Low & $995(21.2)$ & $476(22.3)$ & $18(20.2)$ & $140(18.3)$ & $1,630(21.2)$ & \\
\hline Lowest & $1,381(29.4)$ & $546(25.6)$ & $7(7.9)$ & $187(24.4)$ & $2,121(27.6)$ & \\
\hline \multicolumn{7}{|l|}{ Insurance } \\
\hline Managed care or private insurance & $2,630(56.0)$ & $1,175(55.0)$ & $72(80.9)$ & $453(59.2)$ & $4,331(56.3)$ & \multirow[t]{5}{*}{$<0.0001$} \\
\hline Medicaid/Medicare/government assistance & $1,496(31.9)$ & $637(29.8)$ & $10(11.2)$ & $185(24.2)$ & $2,329(30.3)$ & \\
\hline Tricare/military/Veterans Affairs & $66(1.4)$ & $34(1.6)$ & 0 & $11(1.4)$ & $111(1.4)$ & \\
\hline Not insured & $177(3.8)$ & $118(5.5)$ & $2(2.3)$ & $46(6.0)$ & $343(4.5)$ & \\
\hline Unknown & $326(6.9)$ & $173(8.1)$ & $5(5.6)$ & $70(9.2)$ & $574(7.5)$ & \\
\hline
\end{tabular}

Source: California Cancer Registry. Individuals diagnosed between 1 January 1996 and 31 December 2005

$S D$ standard deviation, $n S E S$ neighborhood socioeconomic status

death was unknown in 52 cases $(2.4 \%)$. The next most common causes of death were infectious and parasitic diseases $(n=37)$ and other respiratory diseases $(n=16)$.

Multivariate survival analysis

Overall survival and leukemia-specific survival analyses were conducted using multivariate Cox regression models that included sex, age group, leukemia type, race/ethnicity, insurance status, and nSES (Table 2). After adjusting for all other variables, HLs and NHBs had a significantly increased adjusted risk of death compared with NHWs.
Age group differences in survival persisted after adjustment for all other variables. Individuals aged 15-29 had increased risk of death compared to children aged $0-14$, with adults aged 30-39 having an even greater increased risk of death. Neighborhood socioeconomic status assignment significantly contributed to risk of death after adjustment for other variables. Trends for increasing risk of mortality with declining nSES were significant (OS: $p=0.01$, LSS: $p=0.007$; Table 2). The combined not insured/unknown insurance status group also had significantly increased mortality risk after adjustment for all other variables. 
Table 2 Multivariate hazard ratios comparing overall survival (OS) and lymphoid/hematopoietic cancer-specific survival (LSS) using Cox proportional hazards model

\begin{tabular}{|c|c|c|c|c|}
\hline \multirow[t]{2}{*}{ Characteristic } & \multicolumn{2}{|l|}{ OS } & \multicolumn{2}{|l|}{ LSS } \\
\hline & HR $(95 \%$ CI $)$ & $p$ & HR $(95 \% \mathrm{CI})$ & $p$ \\
\hline \multicolumn{5}{|l|}{ Age group } \\
\hline $0-14$ & 1.00 (Ref) & - & $1.00($ Ref $)$ & - \\
\hline $15-29$ & $2.65(2.38-2.95)^{*}$ & $<0.0001$ & $2.68(2.39-3.00)^{*}$ & $<0.0001$ \\
\hline $30-39$ & $2.91(2.58-3.29)^{*}$ & $<0.0001$ & $3.01(2.65-3.42)^{*}$ & $<0.0001$ \\
\hline \multicolumn{5}{|l|}{ Race/ethnicity } \\
\hline Non-Hispanic White & $1.00(\operatorname{Ref})$ & - & 1.00 (Ref) & - \\
\hline Non-Hispanic Black & $1.47(1.22-1.76)^{*}$ & $<0.0001$ & $1.56(1.29-1.88)^{*}$ & $<0.0001$ \\
\hline Hispanic/Latino & $1.22(1.09-1.36)^{*}$ & 0.0003 & $1.22(1.09-1.37)^{*}$ & 0.0005 \\
\hline Asian/Pacific Islander & $1.18(1.02-1.37)^{*}$ & 0.0311 & $1.20(1.02-1.41)^{*}$ & 0.0241 \\
\hline \multicolumn{5}{|l|}{ Leukemia type } \\
\hline ALL & 1.00 (Ref) & - & 1.00 (Ref) & - \\
\hline AML & $1.46(1.32-1.61)^{*}$ & $<0.0001$ & $1.44(1.30-1.60)^{*}$ & $<0.0001$ \\
\hline CML & $0.64(0.55-0.75)^{*}$ & $<0.0001$ & $0.62(0.52-0.73)^{*}$ & $<0.0001$ \\
\hline \multicolumn{5}{|l|}{ nSES } \\
\hline Highest & $1.00(\operatorname{Ref}) \dagger$ & - & $1.00(\operatorname{Ref}) \dagger$ & - \\
\hline High & $1.10(0.94-1.29)$ & 0.2374 & $1.18(0.99-1.39)^{*}$ & 0.0591 \\
\hline Middle & $1.19(1.02-1.39)^{*}$ & 0.0298 & $1.24(1.05-1.47)^{*}$ & 0.0110 \\
\hline Low & $1.20(1.03-1.40)^{*}$ & 0.0215 & $1.27(1.07-1.49)^{*}$ & 0.0055 \\
\hline Lowest & $1.31(1.13-1.54)^{*}$ & 0.0006 & $1.37(1.16-1.61)^{*}$ & 0.0002 \\
\hline \multicolumn{5}{|l|}{ Insurance } \\
\hline Any & 1.00 (Ref) & - & $1.00(\operatorname{Ref})$ & - \\
\hline None/unknown & $1.31(1.16-1.47)^{*}$ & 0.0001 & $1.27(1.12-1.44)^{*}$ & 0.0002 \\
\hline
\end{tabular}

Source: California Cancer Registry. Individuals diagnosed between January 1, 1996-December 31, 2005

nSES neighborhood socioeconomic status, $O S$ overall survival, $H R$ hazard ratio, $C I$ confidence interval, Ref reference

All hazard ratios are fully adjusted for the other variables in the model, in addition to gender and diagnostic year

Results for individuals with race/ethnicity other than what is listed $(n=63)$ are suppressed from output

$O S: n=7,597$, with 5,410 observations censored; $L S S: n=7,597$, with 5,613 observations censored

* Significant at $p<0.05$; $\dagger$ significant for trend, $p<0.05$

Age-specific multivariate survival analysis

Multivariate survival analyses were repeated for each of the three age groups (Table 3). As overall survival and leukemia-specific survival rates were quite similar, only OS rates are reported. Adjusted hazard ratios differed between the four race/ethnicity groups, with a wider spread for the group aged 0-14 than the older groups. In children, larger adjusted HRs were evident for AML and CML when compared to ALL. For individuals aged 30-39, a diagnosis of AML or CML was associated with a lower risk of death than a diagnosis of ALL. Only adjusted HRs for nSES maintained a significant gradient for AYAs aged 30-39 ( $p$ value for trend $=0.02$ ). After controlling for other risk factors, not having insurance or having unknown insurance status significantly increased risk of death in subjects aged 0-14 $(p<0.001)$ and 15-29 ( $p=0.03)$, but was not significant in those aged 30-39 $(p=0.119)$.
Race/ethnicity-specific multivariate survival analysis

Multivariate survival analyses stratified by the four major race/ethnicity groups demonstrated its importance as an effect modifier of nSES (Table 4). Survival was shorter for each decreasing nSES quintile for NHWs, and this trend was statistically significant $(p<0.05)$. Although the analyses showed that survival was worse for NHBs in the lower nSES groups (second poorest nSES-adjusted HR: 1.99, 95\% CI: 0.78-5.04; poorest nSES-adjusted HR: 2.23, 95\% CI: 0.87-5.68), the adjusted hazard ratios were not statistically significant, which may be due to low overall numbers for this group. Asian/Pacific Islanders residing in low nSES neighborhoods also had significantly worse survival than those residing in the highest nSES neighborhoods (poorest nSES-adjusted HR: 1.65, 95\% CI: 1.06-2.56). No significant difference among neighborhood nSES on survival was observed for HLs. 
Fig. 1 Kaplan-Meier univariate overall survival curves for California leukemia cases aged 0-39 diagnosed from 1996 to 2005 modified by: a age group, $\mathbf{b}$ insurance status at diagnosis, $\mathbf{c}$ race/ethnicity, and d neighborhood socioeconomic status (nSES)
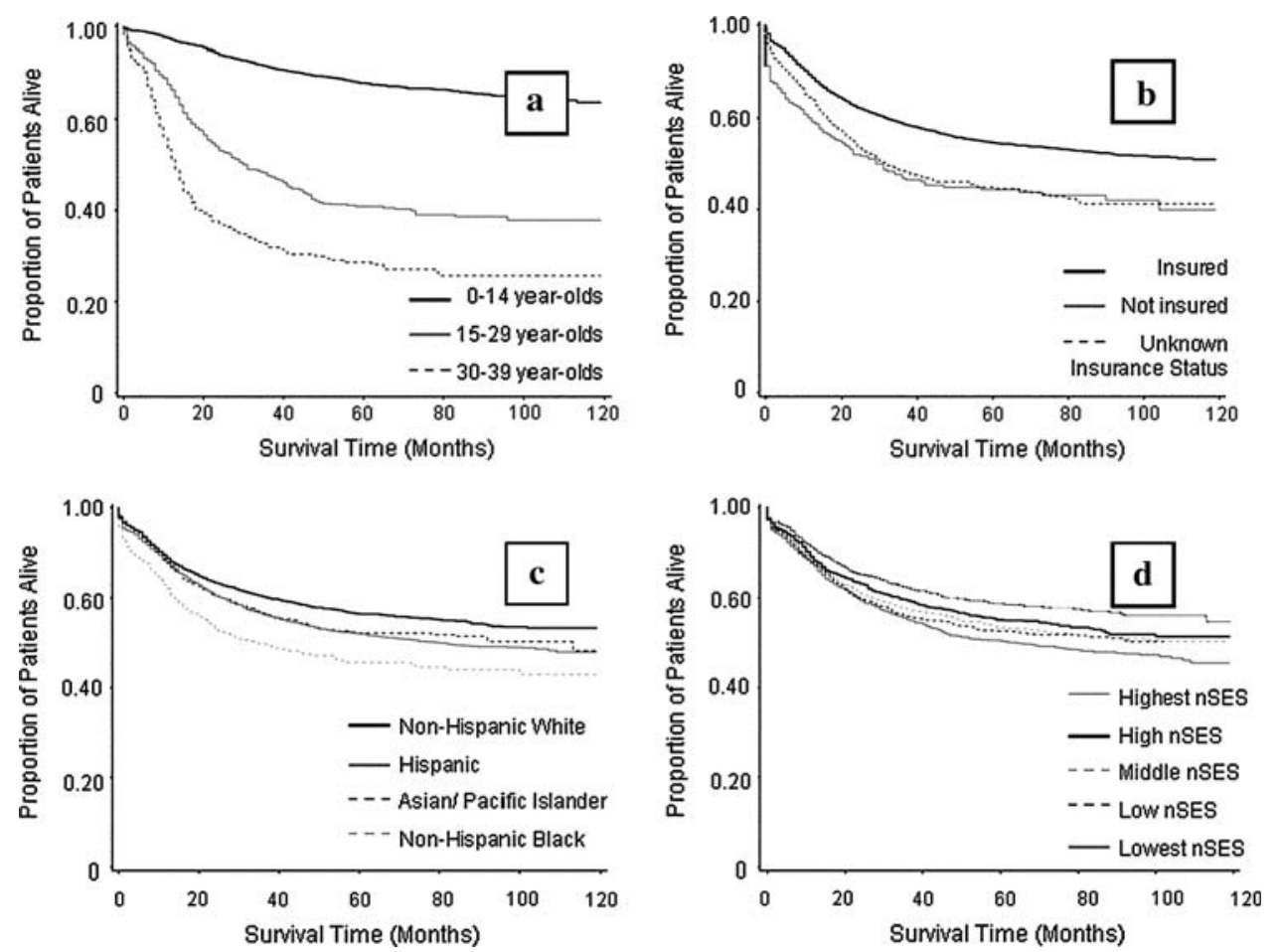

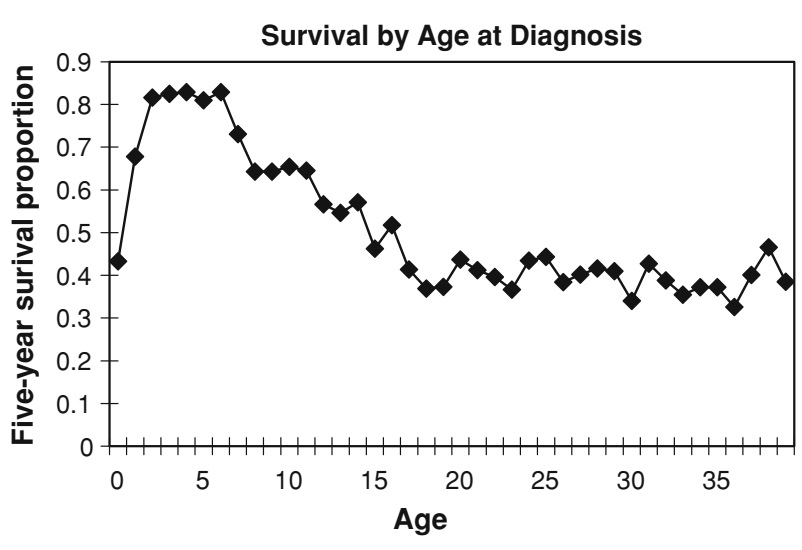

Fig. 2 Five-year survival proportion by each year of age at diagnosis

Leukemia type-specific multivariate survival analysis

Multivariate survival analyses were repeated for each of the three main diagnostic types (Table 5). Subjects diagnosed with ALL between the age of 30 and 39 had 6.94 times higher hazard of death (95\% CI: 5.91-8.14) and subjects aged 15-29 had 3.90 times higher hazard of death (95\% CI: 3.40-4.48) than the youngest age group, after adjusting for other risk factors. By race/ethnicity, nonHispanic blacks with ALL had the largest adjusted HR: 1.70 (95\% CI: $1.27-2.28$ ). Subjects with ALL ( $p$ value for trend $=0.025$ ) and subjects with CML ( $p$ value for trend $=0.031$ ) displayed significant gradients in the relationship between nSES and mortality. For CML, adjusted hazard ratios were higher at every lower quintile of nSES (highest nSES quintile $=$ reference group; second highest nSES HR: 1.25, 95\% CI: 0.74-2.11; middle nSES HR: 1.58, 95\% CI: 0.95-2.63; second poorest nSES HR: 1.78, 95\% CI: 1.06-2.99; poorest nSES HR: 2.10, 95\% CI: 1.27 3.49). Not having insurance or having unknown insurance status contributed to worse survival for subjects with ALL (adjusted HR: 1.37, 95\% CI: 1.16-1.62) and AML (adjusted HR: 1.31, 95\% CI: 1.09-1.57), but not for those with CML.

\section{Discussion}

Our study demonstrated pronounced lower overall survival in young individuals with leukemia living in lower-SES neighborhoods, in adolescents and young adults when compared to children, in NHBs and HLs when compared to NHWs, and in patients uninsured at the time of diagnosis. Further, stratified survival analyses indicated a socioeconomic gradient in survival that was observed only in 30-39 year-old patients, those diagnosed with CML, and NHW patients.

The rather drastic differences we found in survival for ALL among age groups have been previously documented, with worse survival for adolescents aged 15-19 than for children [25]. We found that survival differences also varied among the four major race/ethnicities in California, with NHBs faring the worst for both ALL and AML. The gradient we found between area-level SES and survival has been shown in other registry-based studies of cancer, 
Table 3 Multivariate hazard ratios comparing overall survival (OS) among age groups using Cox proportional hazards model

\begin{tabular}{|c|c|c|c|c|c|c|c|c|c|}
\hline \multirow[t]{3}{*}{ Characteristic } & \multicolumn{9}{|c|}{ Age group } \\
\hline & \multicolumn{3}{|l|}{$0-14$} & \multicolumn{3}{|l|}{$15-29$} & \multicolumn{3}{|l|}{$30-39$} \\
\hline & $n$ & HR & $95 \% \mathrm{CI}$ & $n$ & HR & $95 \% \mathrm{CI}$ & $n$ & HR & $95 \% \mathrm{CI}$ \\
\hline \multicolumn{10}{|l|}{ Race/ethnicity } \\
\hline Non-Hispanic white & 1,379 & 1.00 (Ref) & - & 617 & 1.00 (Ref) & - & 693 & 1.00 (Ref) & - \\
\hline Non-Hispanic black & 178 & $1.54 *$ & $(1.12-2.13)$ & 98 & $1.48^{*}$ & $(1.08-2.03)$ & 112 & 1.26 & $(0.93-1.72)$ \\
\hline Hispanic/Latino & 2,178 & $1.34 *$ & $(1.12-1.63)$ & 936 & 1.13 & $(0.94-1.36)$ & 646 & 1.19 & $(0.98-1.43)$ \\
\hline Asian/Pacific Islander & 385 & 1.19 & $(0.91-1.56)$ & 191 & 1.17 & $(0.90-1.52)$ & 201 & 1.11 & $(0.86-1.44)$ \\
\hline \multicolumn{10}{|l|}{ Leukemia type } \\
\hline ALL & 3,409 & 1.00 (Ref) & - & 877 & 1.00 (Ref) & - & 409 & 1.00 (Ref) & - \\
\hline AML & 675 & $3.59 *$ & $(3.08-4.19)$ & 700 & 1.17 & $(1.00-1.36)$ & 762 & $0.69 *$ & $(0.58-0.81)$ \\
\hline CML & 72 & $2.97 *$ & $(1.96-4.49)$ & 272 & $0.60 *$ & $(0.47-0.77)$ & 421 & $0.32 *$ & $(0.26-0.40)$ \\
\hline \multicolumn{10}{|l|}{ nSES } \\
\hline Highest & 669 & 1.00 (Ref) & - & 237 & 1.00 (Ref) & - & 283 & $1.00^{\dagger}($ Ref $)$ & - \\
\hline High & 677 & 1.15 & $(0.88-1.50)$ & 321 & 0.86 & $(0.65-1.14)$ & 326 & $1.33^{*}$ & $(1.01-1.76)$ \\
\hline Middle & 747 & 0.99 & $(0.75-1.30)$ & 350 & 1.11 & $(0.85-1.45)$ & 324 & $1.52 *$ & $(1.15-2.01)$ \\
\hline Low & 877 & 1.09 & $(0.84-1.43)$ & 321 & 0.99 & $(0.75-1.30)$ & 328 & $1.48^{*}$ & $(1.12-1.95)$ \\
\hline Lowest & 1,118 & 1.13 & $(0.86-1.47)$ & 533 & 1.22 & $(0.93-1.60)$ & 400 & $1.55^{*}$ & $(1.18-2.05)$ \\
\hline \multicolumn{10}{|l|}{ Insurance } \\
\hline Any & 3,797 & 1.00 (Ref) & - & 1,542 & 1.00 (Ref) & - & 1,430 & 1.00 (Ref) & - \\
\hline None/unknown & 361 & $1.56^{*}$ & $(1.26-1.94)$ & 315 & $1.24^{*}$ & $(1.02-1.49)$ & 241 & 1.18 & $(0.96-1.47)$ \\
\hline \multicolumn{10}{|l|}{ Treatment hospital type } \\
\hline Other & 1,346 & 1.00 (Ref) & - & 1,397 & 1.00 (Ref) & - & 1,406 & 1.00 (Ref) & - \\
\hline Pediatric hospital & 1,900 & 1.01 & $(0.85-1.18)$ & 165 & 1.01 & $(0.77-1.31)$ & 0 & - & - \\
\hline Pediatric affiliate hospital & 910 & 0.91 & $(0.74-1.11)$ & 287 & 0.87 & $(0.70-1.07)$ & 186 & 0.95 & $(0.75-1.21)$ \\
\hline
\end{tabular}

Source: California Cancer Registry. Individuals diagnosed between 1 January 1996 and 31 December 2005

All hazard ratios are fully adjusted for the other variables in the model, in addition to gender and diagnostic year

Results for individuals with race/ethnicity other than what is listed $(n=63)$ are suppressed from output

$n S E S$ neighborhood socioeconomic status, $O S$ overall survival, $H R$ hazard ratio, $C I$ confidence interval, Ref reference

OS: $n=7,597$, with 5,410 observations censored

* Significant at $p<0.05 ;{ }^{\dagger}$ significant for trend, $p<0.05$

including melanoma [26] and pancreatic cancer [27]. However, the nSES gradient in our study was significant only in NHWs. Our study consisted of one of the largest sample sizes of API patients with leukemia, and it is noteworthy that there did not appear to be a consistent relationship between nSES and survival in this race/ethnic group.

In addition, the lack of an nSES gradient in survival among Hispanics/Latinos was surprising and raises many questions. Of the cases residing in the lowest SES neighborhoods at diagnosis, $80.5 \%$ were $\mathrm{HL}$, with most $\mathrm{HL}$ patients residing in the lowest $(45.4 \%)$ or second lowest nSES (24.2\%) areas. A report on state-based variations in access to care and use of preventive services found that the percentage of individuals in California who obtained a routine checkup in 1997-2004 compared to 1991-1996 had declined significantly for HLs, but risen significantly for
NHWs [28]. Thus, some of the survival disparity observed for HLs may reflect restricted access to care for this ethnic group, regardless of nSES. Future research should consider possible barriers to care that the current study was unable to determine, such as generational status and primary language spoken at home, which may impact health-seeking behavior in HLs [29]. The existing nSES gradient for NHWs found in the current study suggests, however, that a delivery gap may indeed persist for some young individuals with leukemia, experienced most acutely by older adolescents and young adults.

From our analysis alone, we could not determine whether there are true biological differences for young HL patients with leukemia compared to other racial/ethnic groups or whether even bigger differences exist with aforementioned variables such as access to treatment, trial participation, and education. Aplenc et al. [2] demonstrated 
Table 4 Multivariate hazard ratios comparing overall survival among race/ethnicities using Cox proportional hazards model

\begin{tabular}{|c|c|c|c|c|c|c|c|c|c|c|c|c|}
\hline \multirow[t]{3}{*}{ Characteristic } & \multicolumn{12}{|c|}{ Race/ethnicity } \\
\hline & \multicolumn{3}{|c|}{ Non-Hispanic white } & \multicolumn{3}{|c|}{ Non-Hispanic black } & \multicolumn{3}{|c|}{ Hispanic/Latino } & \multicolumn{3}{|c|}{ Asian/Pacific Islander } \\
\hline & $n$ & HR & $95 \% \mathrm{CI}$ & $n$ & HR & $95 \% \mathrm{CI}$ & $n$ & HR & $95 \% \mathrm{CI}$ & $n$ & HR & $95 \% \mathrm{CI}$ \\
\hline \multicolumn{13}{|l|}{ Age group } \\
\hline $0-14$ & 1,377 & 1.00 (Ref) & - & 178 & $1.00(\operatorname{Ref})$ & - & 2,178 & $\begin{array}{l}1.00 \\
\quad(\operatorname{Ref})\end{array}$ & - & 385 & $\begin{array}{l}1.00 \\
\quad(\operatorname{Ref})\end{array}$ & - \\
\hline $15-29$ & 615 & $2.68^{*}$ & $(2.19-3.28)$ & 97 & $2.03 *$ & $(1.07-3.86)$ & 933 & $2.71 *$ & $(2.34-3.14)$ & 191 & $2.54 *$ & $(1.79-3.62)$ \\
\hline $30-39$ & 647 & $2.80 *$ & $(2.26-3.48)$ & 106 & $2.40 *$ & $(1.58-3.63)$ & 633 & $3.25^{*}$ & $(2.74-3.86)$ & 194 & $2.64 *$ & $(1.82-3.85)$ \\
\hline \multicolumn{13}{|l|}{ Leukemia type } \\
\hline ALL & 1,529 & 1.00 (Ref) & - & 178 & 1.00 (Ref) & - & 2,542 & $\begin{array}{l}1.00 \\
\quad(\operatorname{Ref})\end{array}$ & - & 408 & $\begin{array}{l}1.00 \\
\quad(\operatorname{Ref})\end{array}$ & - \\
\hline AML & 810 & $1.56^{*}$ & $(1.30-1.87)$ & 149 & $1.55^{*}$ & $(1.07-2.26)$ & 915 & $1.40 *$ & $(1.22-1.61)$ & 252 & $1.35^{*}$ & $(0.98-1.87)$ \\
\hline CML & 300 & $0.68^{*}$ & $(0.52-0.90)$ & 54 & $0.47 *$ & $(0.26-0.86)$ & 287 & $0.62 *$ & $(0.48-0.78)$ & 110 & 0.72 & $(0.47-1.13)$ \\
\hline \multicolumn{13}{|l|}{ nSES } \\
\hline Highest & 711 & $1.00^{\dagger}(\mathrm{Ref})$ & - & 23 & 1.00 (Ref) & - & 193 & $\begin{array}{l}1.00 \\
\quad(\operatorname{Ref})\end{array}$ & - & 214 & $\begin{array}{l}1.00 \\
\quad(\operatorname{Ref})\end{array}$ & - \\
\hline High & 680 & 1.17 & $(0.94-1.45)$ & 63 & 1.77 & $(0.66-4.73)$ & 363 & 0.92 & $(0.67-1.26)$ & 190 & 0.97 & $(0.65-1.46)$ \\
\hline Middle & 593 & $1.26^{*}$ & (1.01-1.57) & 86 & 1.53 & $(0.59-3.96)$ & 580 & 0.88 & $(0.66-1.19)$ & 135 & 1.50 & $(1.00-2.24)$ \\
\hline Low & 435 & $1.34 *$ & $(1.06-1.70)$ & 119 & 1.99 & $(0.78-5.04)$ & 904 & 0.87 & $(0.66-1.19)$ & 138 & 1.20 & $(0.79-1.82)$ \\
\hline Lowest & 220 & $1.38^{*}$ & $(1.04-1.70)$ & 90 & 2.23 & $(0.87-5.68)$ & 1,704 & 0.98 & $(0.75-1.28)$ & 93 & $1.65^{*}$ & $(1.06-2.56)$ \\
\hline \multicolumn{13}{|l|}{ Insurance } \\
\hline Any & 2,453 & $1.00(\operatorname{Ref})$ & - & 324 & 1.00 (Ref) & - & 3,158 & $\begin{array}{l}1.00 \\
\quad(\operatorname{Ref})\end{array}$ & - & 701 & $\begin{array}{l}1.00 \\
\text { (Ref) }\end{array}$ & - \\
\hline $\begin{array}{l}\text { None/ } \\
\text { unknown }\end{array}$ & 186 & $1.54^{*}$ & $(1.20-1.98)$ & 57 & $1.69 *$ & (1.09-2.63) & 586 & $1.21 *$ & $(1.04-1.41)$ & 69 & 1.42 & $(0.94-2.13)$ \\
\hline
\end{tabular}

Source: California Cancer Registry. Individuals diagnosed between 1 January 1996 and 31 December 2005

All hazard ratios are fully adjusted for the other variables in the model, in addition to gender and diagnostic year

Results for individuals with race/ethnicity other than what is listed $(n=63)$ are suppressed from output

OS: $n=7,597$, with 5,410 observations censored

nSES neighborhood socioeconomic status, $O S$ overall survival, $H R$ hazard ratio, $C I$ confidence interval, Ref reference

* Significant at $p<0.05 ;{ }^{\dagger}$ significant for trend, $p<0.05$

that African American and Hispanic children with AML had worse OS rates than NHWs in a randomized, controlled clinical trial. Further, a cytogenetic study found a significantly lower frequency of TEL-AML1 translocations (a polymorphism thought to be protective) in HL when compared to NHW children with B-cell ALL [30].

The age group survival disparity we noted has been found in other cancers in the AYA group, along with a lack of measurable progress in survival improvements in 15- to 29 -year-olds relative to all other age groups [4, 15]. Reasons may include the lower rates of enrollment for older teenagers in clinical trials and studies than younger children $[4,31]$. During the 1990 s, only $10-20 \%$ of 15 - to 19 year-olds and fewer than $2 \%$ of 20 - to 29 -year-olds with cancer participated in cancer clinical trials in the United States, compared with 55-65\% of 0- to 14-year-olds [32].

Whether a patient enrolls on a clinical trial or not, 15- to 29 -year-old patients are less likely to see providers from research institutions, which may contribute to worse outcomes [31], and AYAs are often put on adult rather than pediatric treatment regimens, which may ultimately be less effective [33-36]. A recently conducted trial in which the ALL-96 pediatric protocol was administered to 35 adolescents (15- to 18-year-old) and 46 young adults (19- to 30-year-old) found that despite a slight increase in hematologic toxicity, the results were comparable for both groups, warranting usage of the more effective protocol in these older age groups [37].

Although our study covers an ethnically diverse population, which may be appropriate for comparison to other geographic areas, there are some state-specific factors that may be unique to our population. Adolescents and young adults age out of pediatric hospital systems, which provide care only for individuals aged 21 and under [38]. Individuals under the age of 21 in California are eligible for health coverage plans to supplement Medicaid, such as the 
Table 5 Multivariate hazard ratios comparing overall survival among leukemia types using Cox proportional hazards model

\begin{tabular}{|c|c|c|c|c|c|c|c|c|c|}
\hline \multirow[t]{3}{*}{ Characteristic } & \multicolumn{9}{|c|}{ Leukemia type } \\
\hline & \multicolumn{3}{|l|}{ ALL } & \multicolumn{3}{|l|}{ AML } & \multicolumn{3}{|c|}{ CML } \\
\hline & $n$ & HR & $95 \%$ CI & $n$ & HR & $95 \% \mathrm{CI}$ & $n$ & HR & $95 \% \mathrm{CI}$ \\
\hline \multicolumn{10}{|l|}{ Age group } \\
\hline $0-14$ & 3,409 & 1.00 (Ref) & - & 675 & 1.00 (Ref) & - & 72 & 1.00 (Ref) & - \\
\hline $15-29$ & 877 & $3.90 *$ & $(3.40-4.48)$ & 700 & $1.25^{*}$ & $(1.06-1.48)$ & 272 & 0.77 & $(0.48-1.22)$ \\
\hline $30-39$ & 409 & $6.94 *$ & $(5.91-8.14)$ & 762 & $1.24 *$ & $(1.05-1.46)$ & 421 & 0.71 & $(0.46-1.11)$ \\
\hline \multicolumn{10}{|l|}{ Race/ethnicity } \\
\hline Non-Hispanic white & 1,529 & 1.00 (Ref) & - & 810 & 1.00 (Ref) & - & 300 & 1.00 (Ref) & - \\
\hline Non-Hispanic black & 178 & $1.70^{*}$ & $(1.27-2.28)$ & 149 & $1.36^{*}$ & $(1.06-1.76)$ & 54 & 0.91 & $(0.52-1.59)$ \\
\hline Hispanic/Latino & 2,542 & $1.30^{*}$ & $(1.11-1.52)$ & 915 & 1.14 & $(0.97-1.35)$ & 287 & 1.24 & $(0.89-1.75)$ \\
\hline Asian/Pacific Islander & 408 & 1.26 & $(0.99-1.60)$ & 252 & 1.02 & $(0.82-1.28)$ & 110 & 1.45 & $(0.97-2.18)$ \\
\hline \multicolumn{10}{|l|}{ nSES } \\
\hline Highest & 721 & $1.00^{\dagger}$ (Ref) & - & 319 & 1.00 (Ref) & - & 125 & $1.00^{\dagger}$ (Ref) & - \\
\hline High & 768 & 1.18 & $(0.93-1.48)$ & 381 & 1.01 & $(0.79-1.28)$ & 157 & 1.25 & $(0.74-2.11)$ \\
\hline Middle & 830 & 1.06 & $(0.83-1.34)$ & 415 & 1.26 & $(1.00-1.58)$ & 156 & 1.58 & $(0.95-2.63)$ \\
\hline Low & 995 & 1.11 & $(0.88-1.40)$ & 476 & 1.15 & $(0.91-1.45)$ & 140 & $1.78^{*}$ & $(1.06-2.99)$ \\
\hline Lowest & 1,381 & $1.35^{*}$ & $(1.08-1.69)$ & 546 & 1.10 & $(0.86-1.40)$ & 187 & $2.10^{*}$ & $(1.27-3.49)$ \\
\hline \multicolumn{10}{|l|}{ Insurance } \\
\hline Any & 4,192 & 1.00 (Ref) & - & 1,846 & 1.00 (Ref) & - & 649 & 1.00 (Ref) & - \\
\hline None/unknown & 503 & $1.37 *$ & $(1.16-1.62)$ & 291 & $1.31 *$ & $(1.09-1.57)$ & 119 & 1.00 & $(0.67-1.49)$ \\
\hline
\end{tabular}

Source: California Cancer Registry. Individuals diagnosed between 1 January 1996 and 31 December 2005

All hazard ratios are fully adjusted for the other variables in the model, in addition to gender and diagnostic year Results for individuals with race/ethnicity other than what is listed $(n=63)$ are suppressed from output

OS: $n=7,597$, with 5,410 observations censored

nSES neighborhood socioeconomic status, $O S$ overall survival, $H R$ hazard ratio, $C I$ confidence interval, Ref reference

* Significant at $p<0.05 ;{ }^{\dagger}$ significant for trend, $p<0.05$

California Children Services (CCS), which may improve cancer care and mitigate the relationship we found between nSES and survival. The plan requires that enrollees be cared for by a CCS physician; for CCS patients with cancer, most of the affiliated physicians are pediatric oncologists. Several studies have demonstrated that pediatric oncologists generally follow clinical trial protocols, which tend to improve survival for AYAs when compared to standard adult treatment regimens [33-36]. Thus, it is possible that age-specific insurance availability in California may at least partially explain why we did not find a significant survival gradient with increasing nSES for the 15-29 age group.

\section{Strengths and limitations}

This study is the first to examine age group, race/ethnicity, and neighborhood socioeconomic differences in survival among young leukemia patients from such a large population. The large number of person-years in the CCR allows for high-powered analyses of several factors that might affect survivorship and that may be relevant for young patients with leukemia outside of California.

It is possible that there was a degree of under-ascertainment of death in our study; however, every individual contributed a certain amount of survival time before they either died or were censored and patients were followed up annually. Thus, it is unlikely that patients would have been mistakenly assumed to be alive year after year if they were, in fact, deceased. Any misclassification of outcome that resulted from under-ascertainment of death would have likely biased our findings toward the null, as we would have expected that lower-SES patients would be more likely to die without death ascertainment. If there were more low-SES patients who had died but had been classified as censored, then it is possible that we underestimated the relationship between lower SES and risk of death. Only ten $(0.13 \%)$ of the individuals included in the analysis had an unknown source of last patient follow-up, so this possibility is unlikely.

Additional limitations of this study include some likely misclassification of exposure, particularly with the nSES 
variable used, although we expect the effect to be nondifferential with respect to survival and age groupings. Because nSES was assigned by residence at diagnosis, a portion of the cases may have been assigned a neighborhood score that was not truly representative of their personal overall access to resources. If there is a lot of heterogeneity within block groups, then the probability of exposure misclassification would be increased in our study; however, census blocks are considered relatively homogenous in demographics, with most variation attributable between tracts [39]. Looking at resources on only an aggregate level limits the ability to compare the efficacy of the presence of structural resources (proximity to quality treatment facilities, the ability to be included on a research trial) with individual ability to gain access to those resources. On the other hand, only focusing on individuals ignores the broader issues of neighborhood contextual effects on health, such as community resources for healthy living [40]. Previous studies on incidence and survival based on CCR data have tended to use ecological measures of SES [26, 27, 41, 42]. Evidence suggests, however, that personally reported SES dominates area-level measures and that predictive value can only be gained by including area-level SES when behavioral and biological risk factors are not known [43], as was the case in our study.

The neighborhood SES effects on survival that we documented in some of the sub-groups may be a proxy for the types of treatment options available for individuals in certain low resourced areas, for example, having the option of admittance to a pediatric hospital. Previous research has documented the benefits of treatment for AYA leukemia patients at pediatric hospitals [38]. We did not see any significant protection for treatment at a pediatric hospital or pediatric-affiliated hospital in the group for which we included this variable, individuals aged 0-14. This may be an overly simplistic way to look at treatment hospital type; there may be substantial inter-hospital variability with respect to treatment success beyond whether or not the institution specializes in pediatrics, and registry analyses might not be the best method to address these questions.

\section{Conclusions}

Our study demonstrated the importance of age group, race/ ethnicity, nSES, and insurance status on survival of young people with leukemia. We found that the differences in survival across race/ethnicities and age groups persisted after adjustment for nSES and insurance status, which suggests the possibility of important biological differences across these groups. In addition, the dose-response gradient we observed between nSES and survival warrants further investigation into healthcare access and clinical trial participation. Future research is needed to address the survival disparities observed by focusing on variables relevant to children, adolescents, and young adults with cancer, as outlined by the National Cancer Institute and Lance Armstrong Foundation's joint Adolescent and Young Adult Oncology Progress Review Group [38]. We encourage future studies to further explore both individualand neighborhood-level socioeconomic barriers and to examine access to care for diverse racial/ethnic AYA populations.

Acknowledgments This work was supported by the Lon V. Smith Foundation grant LVSF-41027, 42344, and 38340; and the Kids, Adolescents, and Young Adults Cancer (KAYAC) Research Program Gift Fund. The authors would like to especially thank Jason A. Zell, $\mathrm{MD}, \mathrm{MPH}$, of the University of California, Irvine, for assistance with figures and tables; Rebecca A. Morris, MSG, MPH of the CHOC Children's Hospital, for revisions to earlier versions of the manuscript; as well as two anonymous reviewers for their suggestions. Cancer incidence data used in this study under subcontract No. 050N8707-S1527 with the Public Health Institute, State of California, was supported by the California Department of Health Services as part of the statewide cancer reporting program mandated by California Health and Safety Code Sections 103875 and 103885; the National Cancer Institute Surveillance, Epidemiology and End Results Program; and the Centers for Disease Control and Prevention National Program of Cancer Registries.

Disclaimer The ideas and opinions expressed herein are those of the authors, and endorsement by the State of California, Department of Health Services, the National Cancer Institute, the Centers for Disease Control and Prevention, and/or the Genetic Epidemiology Research Institute of the University of California, Irvine is not intended, nor should it be inferred. The authors indicated no potential conflicts of interest.

Open Access This article is distributed under the terms of the Creative Commons Attribution Noncommercial License which permits any noncommercial use, distribution, and reproduction in any medium, provided the original author(s) and source are credited.

\section{References}

1. Campleman SL, Wright WE (2004) Childhood cancer in California 1988 to 1999 volume II: ages 15-19. California Department of Health Services, Cancer Surveillance Section, Sacramento

2. Aplenc R, Alonzo TA, Gerbing RB et al (2006) Ethnicity and survival in childhood acute myeloid leukemia: a report from the Children's Oncology Group. Blood 108(1):74-80. doi:10.1182/ blood-2005-10-4004

3. Bhatia S, Sather HN, Heerema NA, Trigg ME, Gaynon PS, Robison LL (2002) Racial and ethnic differences in survival of children with acute lymphoblastic leukemia. Blood 100(6):19571964. doi:10.1182/blood-2002-02-0395

4. Bleyer A, Viny A, Barr R (2006) Cancer in 15- to 29-year-olds by primary site. Oncologist 11(6):590-601. doi:10.1634/the oncologist.11-6-590

5. Ford JG, Howerton MW, Lai GY et al (2008) Barriers to recruiting underrepresented populations to cancer clinical trials: a 
systematic review. Cancer 112(2):228-242. doi:10.1002/cncr. 23157

6. Fagundes EM, Rocha V, Glória AB et al (2006) De novo acute myeloid leukemia in adults younger than 60 years of age: socioeconomic aspects and treatment results in a Brazilian university center. Leuk Lymphoma 47(8):1557-1564. doi:10.1080/ 10428190600627055

7. Zebrack B (2009) Information and service needs for young adult cancer survivors. Support Care Cancer 17(4):349-357. doi: 10.1007/s00520-008-0469-2

8. Ward E, Halpern M, Schrag N et al (2008) Association of insurance with cancer care utilization and outcomes. CA Cancer J Clin 58(1):9-31. doi:10.3322/CA.2007.0011

9. Evans GW (2004) The environment of childhood poverty. Am Psychol 59(2):77-92. doi:10.1037/0003-066X.59.2.77

10. Galobardes B, Shaw M, Lawlor DA, Smith GD, Lynch J (2006) Indicators of socioeconomic position. In: Oakes JM, Kaufman JS (eds) Methods in social epidemiology. Jossey-Bass, San Francisco, pp 47-85

11. Guidry JJ, Aday LA, Zhang D, Winn RJ (1998) Cost considerations as potential barriers to cancer treatment. Cancer Pract 6(3):182-187. doi:10.1046/j.1523-5394.1998.006003182.x

12. Ries L, Melbert D, Krapcho $M$ et al (2008) SEER cancer statistics review, 1975-2005. National Cancer Institute, Bethesda

13. Pulte D, Gondos A, Brenner H (2009) Improvement in survival in younger patients with acute lymphoblastic leukemia from the 1980 s to the early 21 st century. Blood 113(7):1408-1411. doi: 10.1182/blood-2008-06-164863

14. Birch JM, Pang D, Alston RD et al (2008) Survival from cancer in teenagers and young adults in England, 1979-2003. Br J Cancer 99(5):830-835. doi:10.1038/sj.bjc.6604460

15. Feltbower RG, McNally RJ, Kinsey SE et al (2009) Epidemiology of leukaemia and lymphoma in children and young adults from the north of England, 1990-2002. Eur J Cancer 45(3):420427. doi:10.1016/j.ejca.2008.09.020

16. Cancer Surveillance Section (1997) Cancer reporting in California: standards for automated reporting. California cancer reporting system standards, vol II. California Department of Health Services, Cancer Surveillance Section, Sacramento

17. Cancer Surveillance Section (1997) Cancer reporting in California: data standard for regional registries and California Cancer Registry. California cancer reporting system standards, vol III. California Department of Health Services, Cancer Surveillance Section, Sacramento

18. Cancer Surveillance Section (1997) Abstract and coding procedures for hospitals. California cancer reporting system standards, vol I. California Department of Health Services, Cancer Surveillance Section, Sacramento

19. Cancer Surveillance Section (1997) Reporting procedures for physicians. California cancer reporting system standards, vol IV. California Department of Health Services, Cancer Surveillance Section, Sacramento

20. California Cancer Registry (2008) How complete are California Cancer Registry Data? Available from: http://www.ccrcal.org/ questions.html

21. Fritz A, Percy C, Jack A et al (2000) International classification of diseases for oncology (ICD-O), 3rd edn. World Health Organization, Geneva

22. Yost K, Perkins C, Cohen R, Morris C, Wright W (2001) Socioeconomic status and breast cancer incidence in California for different race/ethnic groups. Cancer Causes Control 12(8):703-711. doi:10.1023/A:1011240019516

23. World Health Organization (2005) The international statistical classification of diseases and related health problems, 10th revision. World Health Organization, Geneva
24. Lightfoot T (2005) Aetiology of childhood leukemia. Bioelectromagnetics 7(Suppl):S5-S11. doi:10.1002/bem.20140

25. Desandes E (2007) Survival from adolescent cancer. Cancer Treat Rev 33(7):609-615. doi:10.1016/j.ctrv.2006.12.007

26. Zell JA, Cinar P, Mobasher M, Ziogas A, Meyskens FL Jr, Anton-Culver H (2008) Survival for patients with invasive cutaneous melanoma among ethnic groups: the effects of socioeconomic status and treatment. J Clin Oncol 26(1):66-75. doi: 10.1200/JCO.2007.12.3604

27. Zell JA, Rhee JM, Ziogas A, Lipkin SM, Anton-Culver H (2007) Race, socioeconomic status, treatment, and survival time among pancreatic cancer cases in California. Cancer Epidemiol Biomarkers Prev 16(3):546-552. doi:10.1158/1055-9965.EPI-060893

28. Kang-Kim M, Betancourt JR, Ayanian JZ, Zaslavsky AM, Yucel RM, Weissman JS (2008) Access to care and use of preventive services by Hispanics: state-based variations from 1991 to 2004 . Med Care 46(5):507-515. doi:10.1097/MLR.0b013e31816dd966

29. DuBard CA, Gizlice Z (2008) Language spoken and differences in health status, access to care, and receipt of preventive services among US Hispanics. Am J Public Health 98(11):2021-2028. doi:10.2105/AJPH.2007.119008

30. Aldrich MC, Zhang L, Wiemels JL et al (2006) Cytogenetics of Hispanic and white children with acute lymphoblastic leukemia in California. Cancer Epidemiol Biomarkers Prev 15(3):578-581. doi:10.1158/1055-9965.EPI-05-0833

31. Burke ME, Albritton K, Marina N (2007) Challenges in the recruitment of adolescents and young adults to cancer clinical trials. Cancer 110(11):2385-2393. doi:10.1002/cncr.23060

32. Bleyer WA, Tejeda H, Murphy SB et al (1997) National cancer clinical trials: children have equal access; adolescents do not. J Adolesc Health 21(6):366-373. doi:10.1016/S1054-139X(97) 00110-9

33. Barry E, DeAngelo DJ, Neuberg D et al (2007) Favorable outcome for adolescents with acute lymphoblastic leukemia treated on Dana-Farber Cancer Institute Acute Lymphoblastic Leukemia Consortium Protocols. J Clin Oncol 25(7):813-819. doi: 10.1200/JCO.2006.08.6397

34. Hallböök H, Gustafsson G, Smedmyr B et al (2006) Treatment outcome in young adults and children $>10$ years of age with acute lymphoblastic leukemia in Sweden: a comparison between a pediatric protocol and an adult protocol. Cancer 107(7):15511561. doi: $10.1002 / \mathrm{cncr} .22189$

35. Ramanujachar R, Richards S, Hann I, Webb D (2006) Adolescents with acute lymphoblastic leukaemia: emerging from the shadow of paediatric and adult treatment protocols. Pediatr Blood Cancer 47(6):748-756. doi:10.1002/pbc.20776

36. Stock W, La M, Sanford B et al (2008) What determines the outcomes for adolescents and young adults with acute lymphoblastic leukemia treated on cooperative group protocols? A comparison of Children's Cancer Group and Cancer and Leukemia Group B studies. Blood 112(5):1646-1654. doi:10.1182/ blood-2008-01-130237

37. Ribera JM, Oriol A, Sanz MA et al (2008) Comparison of the results of the treatment of adolescents and young adults with standard-risk acute lymphoblastic leukemia with the Programa Español de Tratamiento en Hematologia pediatric-based protocol ALL-96. J Clin Oncol 26(11):1843-1849. doi:10.1200/JCO. 2007.13.7265

38. Adolescent and Young Adult Oncology Progress Review Group (2006) Closing the gap: research and care imperatives for adolescents and young adults with cancer. Bethesda, MD: Department of Health and Human Services, National Institutes of Health, National Cancer Institute, and the LiveStrong ${ }^{\text {TM }}$ Young Adult Alliance. NIH Publication No. 06-6067 
39. Diez Roux AV, Kiefe CI, Jacobs DR Jr et al (2001) Area characteristics and individual-level socioeconomic position indicators in three population-based epidemiologic studies. Ann Epidemiol 11(6):395-405. doi:10.1016/S1047-2797(01)00221-6

40. Krieger N (2005) Defining and investigating social disparities in cancer: critical issues. Cancer Causes Control 16(1):5-14. doi: 10.1007/s10552-004-1251-5

41. Ou SH, Zell JA, Ziogas A, Anton-Culver H (2007) Prognostic factors for survival of stage I nonsmall cell lung cancer patients: a population-based analysis of 19, 702 stage I patients in the
California Cancer Registry from 1989 to 2003. Cancer 110(7):1532-1541. doi:10.1002/cncr.22938

42. Robbins AS, Whittemore AS, Thom DH (2000) Differences in socioeconomic status and survival among white and black men with prostate cancer. Am J Epidemiol 151(4):409-416

43. Thomas AJ, Eberly LE, Neaton JD, Davey Smith G, Multiple Risk Factor Intervention Trial (MRFIT) Research Group (2007) Census-based and personally reported income measures as long-term risk-adjusted mortality predictors. Public Health 121(12):898-901. doi:10.1016/j.puhe.2007.03.019 